[Radiocarbon, Vol. 21, No. 2, 1979, P. 306-320]

\title{
US GEOLOGICAL SURVEY, RESTON, VIRGINIA, RADIOCARBON DATES XV : MAUNA LOA AND KILAUEA VOLCANOES, HAWAII
}

\author{
M L KELLEY ${ }^{1}$, E C SPIKER ${ }^{1}$, P W LIPMAN ${ }^{2}$, J P LOCKWOOD ${ }^{3}$, \\ R T HOLCOMB ${ }^{4}$, and M RUBIN ${ }^{1}$
}

INTRODUCTION

Ninety-six new ${ }^{14} \mathrm{C}$ dates are reported for carbonized roots and other plant material coll from beneath prehistoric lava flows and ash deposits from Mauna Loa (ML) and Kilauea volcanoes. Before 1976, only 10 flows from these volcanoes had been dated by radiocarbon methods. Collection of dateable material has been facilitated by an improved understanding of the conditions of charcoal formation and preservation beneath basaltic lavas (Lockwood \& Lipman, 1979).

The new dates indicate that the brief recorded history of ML eruptive activity, beginning about 1840, has documented an exceptionally active period. For example, 5 flows from the SW rift zone of ML reached the ocean between 1868 and 1950, but in the preceding 500 yr only 3 flows appear to have reached the sea. In contrast, Kilauea flank eruptions along the $\mathrm{E}$ rift zone appear to have been more extensive before $\sim 200$ yr ago than since that time. The available radiocarbon dates also show that the surface flows on ML tend to be older than those of Kilauea. Flows several thousand yr old are common on ML, but flows more than a few hundred yr old are rare on Kilauea.

Samples are counted in the form of acetylene gas, as previously, and ages are expressed in radiocarbon yr based on the Libby half-life, $5568 \pm$ $30 \mathrm{yr}$, referenced to the $\mathrm{yr}$ AD 1950. The dates are not corrected for isotopic fractionation or natural ${ }^{14} \mathrm{C}$ variations. Errors are stated in terms of one standard deviation based only on the counting statistics and not the analysis of replicate samples from the field. The counting error does not include variable factors such as contamination and isotopic fractionation, which if considered, may increase the confidence interval in some cases by a factor of 2 or 3. Unless otherwise noted, collectors of all samples are members of the US Geological Survey.

\section{Mauna Loa series}

W-4198.

Charcoal fragments along basal aa, at edge of Manuka flow, resting on pumice blanket from Hapaimanu, $\mathrm{N}$ end of prominent kipuka, NW of Pohakuloa, alt $1481 \mathrm{~m}$, Papa quad $\left(19^{\circ} 10.47^{\prime} \mathrm{N}, 155^{\circ} 46.62^{\prime} \mathrm{W}\right)$. Coll 1978 by P W Lipman. Comment (PWL): youngest prehistoric flow along lower middle SW rift zone. This flow is more weathered and supports more mature forest than any historic (as old as 1868) flow on SW rift zone.

\footnotetext{
${ }^{1}$ US Geological Survey, Reston, Virginia 22092

${ }^{2}$ US Geological Survey, Denver, Colorado 80225

${ }^{3}$ US Geological Survey, Hawaiian Volcano Observatory, Hawaii 96718

4 Stanford University, Dept of Geology, Stanford, California 94305
} 
Some trees (Ohia lehua) are as much as $0.5 \mathrm{~m}$ diam, which suggests age of several hundred yr.

W-3843.

Charcoal root fragments from Pahala ash below pahoehoe flow of Kipuka Maunaiu, at head of gully, along contact with Keomoku flow, alt $1050 \mathrm{~m}$, Ohaikea Rd, Kilauea quad $\left(19^{\circ} 24.54^{\prime} \mathrm{N}, 155^{\circ} 20.61^{\prime} \mathrm{W}\right)$. Coll 1977 by P W Lipman. Comment (PWL): this flow is overlain by Keomoku flow unit $(\mathrm{W}-3871)$ dated at $230 \pm 60$.

W-4186.

$220 \pm 60$

Charcoal, beneath thin pahoehoe toes at contact with old Mauna Kea aa around small kipuka, "Red Leg Trail," $5 \mathrm{~km}$ SSW of Pohakuloa cabins, alt $1914 \mathrm{~m}$, Puu Koli quad (19 $\left.42.68^{\prime} \mathrm{N}, 155^{\circ} 33.15^{\prime} \mathrm{W}\right)$. Coll 1978 by J P Lockwood. Comment (JPL): one of younger prehistoric tubefed pahoehoe flows on $\mathrm{N}$ side of NE rift. This may be same spectacular lava-tube flow seen at helicopter launch zone on ML Observatory Rd at $2800 \mathrm{~m}$. Uncharred wood was preserved at margin of flow in this dry area.

W-3871.

$230 \pm 60$

Charcoal roots in ash below Keomoku aa flow, from roadcuts in Kipuka Ki, alt $1281 \mathrm{~m}$, Kilauea quad (19²6.73' N, $\left.155^{\circ} 19.12^{\prime} \mathrm{W}\right)$. Coll 1977 by P W Lipman. Comment (PWL): sample dates youngest major lobe of Keomoku flow units and represents last major prehistoric flow on SE side of ML. Vent is at $3050 \mathrm{~m}$ level on NE rift, and age determination provides key reference point for late prehistoric history of ML. This lobe is stratigraphically above $\mathrm{W}-3843(<200)$ and $\mathrm{W}-4006(290 \pm 70)$, and confirms young age of this Keomoku lobe. According to J P Lockwood, some parts of Keomoku flow sequence are older than Kulani Picrite, which has yielded ages of 420 to $450 \mathrm{yr}, \mathrm{W}-3790$ and -3793 . These ages indicate some significant age difference between lobes mapped as Keomoku lavas by Peterson (1967).

W-4006.

$290 \pm 70$

Charcoal roots in Pahala ash, underlying Kipuka Maunaiu flow, Ohaikea Rd, alt $1095 \mathrm{~m}$, Kilauea quad $\left(19^{\circ} 24.73^{\prime} \mathrm{N}, 155^{\circ} 20.55^{\prime} \mathrm{W}\right)$. Coll 1977 by P W Lipman. Comment (PWL): in reasonable agreement with W-3843 $(<200)$, from below same flow. Confirms young age of this prehistoric flow.

W-4183.

$300 \pm 60$

Charcoal, SW margin of prominent "l" red pahoehoe kipuka on W margin of Kau 1880 flow, alt 2550 m, Kipuka Pakekake quad $\left(19^{\circ} 29.74^{\prime} \mathrm{N}\right.$, $\left.155^{\circ} 26.66^{\prime} \mathrm{W}\right)$. Coll 1978 by J P Lockwood. Comment (JPL): dates a very young prehistoric ML eruption-erupted from vent at $3340 \mathrm{~m}$ on NE rift, $600 \mathrm{~m}$ SE of Pukauahi.

W-4175.

$\mathbf{3 0 0} \pm \mathbf{5 0}$

Charcoal underlying dark gray aa, overlain by 1880 lavas, Kulani MLO Rd, $3 \mathrm{~km} \mathrm{E} \mathrm{of} \mathrm{Telephone} \mathrm{Relay} \mathrm{Sta,} \mathrm{alt} \mathrm{2292m,} \mathrm{Puu} \mathrm{Ulaula} \mathrm{quad}$ 
$\left(19^{\circ} 35.20^{\prime} \mathrm{N}, 155^{\circ} 25.29^{\prime} \mathrm{W}\right)$. Coll 1978 by J P Lockwood. Comment (JPL): vent for this flow is dark, perfectly formed spatter cone, $2 \mathrm{~km} \mathrm{NE}$ of Puu Ulaula (puu 2928m). This cone had previously been dated, erroneously, at AD 1881.

\section{W-4049.}

$420 \pm 70$

Vitreous charcoal from Keomoku Flow-upper "K1," underlying thin outlier of aa, alt $1498 \mathrm{~m}$, Kilauea quad (19०2 $\left.27.78^{\prime} \mathrm{N}, 155^{\circ} 20.80^{\prime} \mathrm{W}\right)$. Coll 1978 by J P Lockwood. Comment (JPL): this flow underlies W-4118 flow $(580 \pm 80)$.

\section{W-3790.}

$420 \pm 70$

Charcoal from thin soil, developed on old spatter, underlying Kulani Picrite, on high-standing dry hillock, Kulani quad $\left(19^{\circ} 33.25^{\prime} \mathrm{N}, 155^{\circ}\right.$ 18.07' W). Coll 1977 by J P Lockwood. Comment (JPL): this sample was coll to compare with $\mathrm{W}-3793$, to see if charcoal saturation by water has any effect on ${ }^{14} \mathrm{C}$ content; apparently it has no effect.

W-3793.

$450 \pm 60$

Charcoal from water-saturated spatter underlying $2 \mathrm{~m}$ of Kulani Picrite, Kulani quad $\left(19^{\circ} 33.12^{\prime}\right.$ N, $\left.155^{\circ} 18.03^{\prime} \mathrm{W}\right)$. Coll 1977 by J P Lockwood.

W-3880.

$$
530 \pm 60
$$

Organic debris and sooty soil directly underlying ropy pahoehoe, Kilauea Crater quad $\left(19^{\circ} 27.80^{\prime} \mathrm{N}, 155^{\circ} 17.07^{\prime} \mathrm{W}\right)$. Coll 1977 by J P Lockwood.

\section{W-4118.}

$$
\mathbf{5 8 0} \pm \mathbf{8 0}
$$

Charcoal from younger phase of "KKl" unit of Keomoku Flow, $875 \mathrm{~m} \mathrm{~N}$ of end of Strip Rd, $300 \mathrm{~m}$ E of Puu Ulaula Trail, alt $2079 \mathrm{~m}$, Puu Ulaula quad $\left(19^{\circ} 30.19^{\prime} \mathrm{N}, 155^{\circ} 23.16^{\prime} \mathrm{W}\right)$. Coll 1978 by J P Lockwood.

\section{W-3898.}

$590 \pm 70$

Charcoal from pocket of baked ash, underlying very young precaldera aa flow, alt 2388, Ainapo Trail, ML quad $\left(19^{\circ} 23.48^{\prime} \mathrm{N}, 155^{\circ}\right.$ $30.92^{\prime}$ W). Coll 1977 by J P Lockwood. Comment (JPL): date is max for formation of present Mokuaweoweo caldera, because flow was erupted from area now blocked by caldera wall.

\section{W-4025.}

$640 \pm 45$

Charcoal fragments at base of Pohina flow, where it rests on Pahala ash at edge of Na Manua Haalou swamp, alt $1774 \mathrm{~m}$, Pun O Keokeo quad $\left(19^{\circ} 13.15^{\prime} \mathrm{N}, 155^{\circ} 37.90^{\prime} \mathrm{W}\right)$. Coll 1977 by P W Lipman. Comment (PWL): flow characterized by abundant large olivine phenocrysts and erupted from young-appearing prehistoric cones at $2900 \mathrm{~m}$ level on SW rift zone; this is youngest flow to reach ocean on S side of ML. 
W-4156.

Charcoal roots from ash at base of Kipuka Nene flow, exposed in pothole along Hilea Gulch, alt $473 \mathrm{~m}$, Punaluu quad $\left(19^{\circ} 09.04^{\prime} \mathrm{N}, 155^{\circ}\right.$ 33.93' W). Coll 1977 by P W Lipman. Comment (PWL): dates youngest pahoehoe flow to reach ocean on Kau side of SW rift zone.

W-4012.

$$
740 \pm 60
$$

Charcoal roots from ash, underlying thin "variable-olivine" pahoehoe flow at Kipuka Nene, coll in Hilea Gulch, alt $305 \mathrm{~m}$, Punaluu quad $\left(19^{\circ} 08.28^{\prime} \mathrm{N}, 155^{\circ} 33.14^{\prime} \mathrm{W}\right)$. Coll 1977 by $\mathrm{P}$ W Lipman. Comment (PWL): together with $890 \pm 60, \mathrm{~W}-4137$, and $740 \pm 60, \mathrm{~W}-4156$, represents last widespread voluminous pahoehoe eruptive activity low on this sector of ML.

W-3879.

Charcoal from flaky roots in Pahala ash, $15 \mathrm{~cm}$ beneath fluid ropy

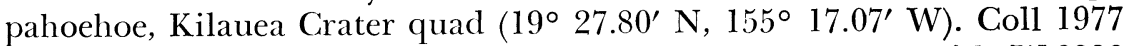
by J P Lockwood. Comment (JPL): reasonable agreement with W-3880 $(530 \pm 60)$, a charred soil sample.

W-4137.

$890 \pm 60$

Charcoal roots from lens of Pahala-like ash, below thin olivine-rich pahoehoe (Kipuka Nene flow), coll in Hilea Gulch, alt $381 \mathrm{~m}$, Punaluu quad $\left(19^{\circ} 08.50^{\prime} \mathrm{N}, 155^{\circ} 33.29^{\prime} \mathrm{W}\right)$. Coll 1977 by P W Lipman. Comment (PWL): together with $740 \pm 60, \mathrm{~W}-4012$ and -4156 , may date 3 related flows of similar appearance or different parts of single eruption sequence.

\section{W-4047.}

$910 \pm 70$

Charcoal under olivine-rich aa flow, alt 1934m, Kipuka Pakekake quad $\left(19^{\circ} 29.25^{\prime} \mathrm{N}, 155^{\circ} 23.19^{\prime} \mathrm{W}\right)$. Coll 1977 by J P Lockwood. Comment (JPL): this is oldest lava flow emanating from a known vent on ML's NE rift zone. Source of flow is $3027 \mathrm{~m}$ cone, $600 \mathrm{~m}$ NE of Puu Ulaula.

W-3910.

$1270 \pm 60$

Charcoal in ash pocket underlying tree mold in fluid tube-fed late prehistoric pahoehoe flow, Ainapo Trail, ML S flank, alt 2277m, ML quad $\left(19^{\circ} 23.33^{\prime} \mathrm{N}, 155^{\circ} 30.66^{\prime}\right.$ W). Coll 1977 by J P Lockwood. Comment (JPL): date is another max of present extent of Mokuaweoweo caldera.

\section{W-3000.}

$1330 \pm 70$

Organic debris and some charcoal, at uppermost part of yellow ash sec, overridden by ML pahoehoe flow, Kaoiki Pali, alt $915 \mathrm{~m}$, Wood Valley quad $\left(19^{\circ} 22.48^{\prime} \mathrm{N}, 155^{\circ} 22.55^{\prime} \mathrm{W}\right)$. Coll 1973 by R I Tilling. Comment (PWL): dates 1 of most recent lavas draped across this sec of Kaoiki fault zone.

W-3858.

$1400 \pm 60$

Carbonized wood fragments (Ohia lehua) in Pahala ash, beneath young prehistoric ML pahoehoe flow, Kaalaala Gulch, alt 457m, Pahala 
quad (19 $\left.13.43^{\prime} \mathrm{N}, 155^{\circ} 27.94^{\prime} \mathrm{W}\right)$. Coll 1977 by P W Lipman. Comment $(\mathrm{PWL})$ : dates 1 of youngest large eruptions to flow $\mathrm{S}$ from summit area; possibly related in time and lithology to W-3850 (1810 \pm 80$)$.

W-3857.

$1470 \pm 60$

Charcoal fragments of different, but unid., plant sp, from same location as W-3858. Coll 1977 by P W Lipman.

W-3850.

$1810 \pm 80$

Charcoal in Pahala ash, overlain by phenocryst-poor ML pahoehoe from SW rift zone, Moaula gulch, alt $137 \mathrm{~m}$, Pahala quad $\left(19^{\circ} 11.23^{\prime} \mathrm{N}\right.$, $155^{\circ} 29.15^{\prime} \mathrm{W}$ ). Coll 1977 by P W Lipman. Comment (PWL): min date for Pahala Ash in type area; probably dates widespread pahoehoe flow sequence, along Hwy 11 to $\mathrm{S}$ for several $\mathrm{km}$, that constitutes last major eruption to extend this far down flank of ML from upper SW rift zone.

\section{W-4116.}

$1840 \pm 60$

Charcoal at contact of pahoehoe/aa thin soil, $1.4 \mathrm{~km}$ below end of

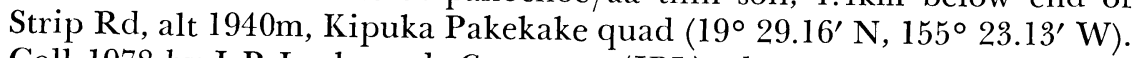
Coll 1978 by J P Lockwood. Comment (JPL): dates very extensive tubefed pahoehoe flow, which is widespread on upper Strip $\mathrm{Rd}$ and lower Puu Ulaula trail.

W-4022.

$1860 \pm 70$

Charcoal fragments at base of plagioclase-phyric aa flow, Punaluu Kahawai, alt $1891 \mathrm{~m}$, Punaluu quad $\left(19^{\circ} 14.94^{\prime} \mathrm{N}, 155^{\circ} 36.83^{\prime} \mathrm{W}\right)$. Coll 1977 by P W Lipman. Comment (PWL): closely related to Ninole flow, $\mathrm{W}-4008(2300 \pm 60)$, erupted from large spatter ramparts on SW rift zone, $1 \mathrm{~km}$ below Sulphur Cone.

W-4163.

$1980 \pm 80$

Charcoal roots in Pahala ash at base of flow, underlying phenocrystpoor aa flow, coll where aa cascaded through small lava tube, Kauhuhuula Gulch, alt $2036 \mathrm{~m}$, Keaiwa Reservoir quad $\left(19^{\circ} 18.02^{\prime} \mathrm{N}, 155^{\circ}\right.$ $33.72^{\prime}$ W). Coll 1977 by P W Lipman. Comment (PWL): dates major vent system at $3350 \mathrm{~m}$ level on ML's SW rift. These vents, which include Sulphur Cone, are among oldest exposed this high on rift zone.

\section{W-4161.}

$2000 \pm 70$

Charcoal fragments from basal Hionamoa flow, a large phenocrystpoor unit, coll among head of Makaka Ravine, alt $2060 \mathrm{~m}$, Keaiwa Reservoir quad $\left(19^{\circ} 17.79^{\prime} \mathrm{N}, 155^{\circ} 34.20^{\prime} \mathrm{W}\right)$. Coll 1977 by P W Lipman. Comment (PWL): in excellent agreement with age on a petrographically similar nearby flow, W-4163 (1980 \pm 80$)$.

\section{W-4017.}

$2010 \pm 70$

Charcoal fragments from Pahala-like ash, underlying weathered phenocryst-poor pahoehoe, Hionamoa Gulch, alt 2074m, Keaiwa quad $\left(19^{\circ} 17.17^{\prime} \mathrm{N}, 155^{\circ} 35.40^{\prime} \mathrm{W}\right)$. Coll 1977 by $\mathrm{P}$ W Lipman. Comment 
(PWL): flow is among oldest exposed at tree line, or above, on ML and provides max age for at least $95 \%$ of rocks exposed on upper parts of $\mathrm{mt}$.

\section{W-4015.}

$2180 \pm 60$

Charred log, base of young aa flow containing large olivine pheno-

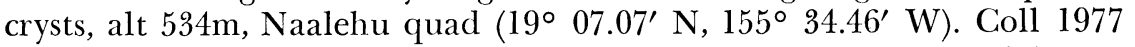
by P W Lipman. Comment (PWL): this flow and similar large olivine aa flow to NE, W-4025 $(640 \pm 50)$ are youngest lava flows to reach ocean between Pahala and Naalehu. These 2 flows were correlated at first; difference in ages is surprise.

W-3876.

$2190 \pm 70$

Charcoal from root underlying NE rift ML aa flow, covering Pahala ash on E extension of Kaoiki fault, Puu Oo Trail, Kilauea Crater quad $\left(19^{\circ} 27.83^{\prime} \mathrm{N}, 155^{\circ} 17.60^{\prime} \mathrm{W}\right)$. Coll 1977 by J P Lockwood.

\section{W.4008.}

$2300 \pm 60$

Carbonized wood fragments in ash at base of plagioclase-phyric Ninole aa flow, one of last flows from this sec of ML to reach ocean, Ninole Gulch, alt $316 \mathrm{~m}$, Punaluu quad $\left(19^{\circ} 09.38^{\prime} \mathrm{N}, 155^{\circ} 32.43^{\prime} \mathrm{W}\right)$. Coll 1977 by P W Lipman.

\section{W-4142.}

$$
2440 \pm 60
$$

Charcoal fragments in Pahala-like ash, base of Ninole aa flow, Ninole Gulch, alt $53 \mathrm{~m}$, Punaluu quad $\left(19^{\circ} 08.60^{\prime} \mathrm{N}, 155^{\circ} 31.32^{\prime} \mathrm{W}\right)$. Coll 1977 by P W Lipman. Comment (PWL): in good agreement with $\mathrm{W}-4008(2300 \pm 60)$ from base of same flow.

\section{W-3836.}

$2830 \pm 60$

Carbonaceous soil below aa of Keomoku flow, alt $1064 \mathrm{~m}$, Kilauea quad $\left(19^{\circ} 24.46^{\prime} \mathrm{N}, 155^{\circ} 20.64^{\prime} \mathrm{W}\right)$. Coll 1977 by P W Lipman. Comment (PWL): anomalous age for unknown reasons. Does not agree with age of $230 \pm 60, \mathrm{~W}-3871$, from below same flow, or $<200, \mathrm{~W}-3843$, and $290 \pm 70$, W-4006, which date underlying Kipuka Maunaiu flow.

\section{W-3845.}

$2880 \pm 70$

Charcoal root fragments from top of Pahala ash below olivine-rich ML pahoehoe flow, where draped over Kaoiki fault scarp, Kaoiki Pali,

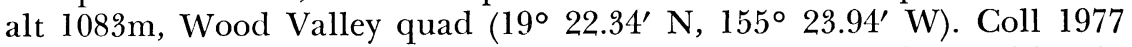
by P W Lipman. Comment (PWL): min date for last major faulting in area.

W-4174.

$2890 \pm 70$

Amorphous sooty material underlying Panaewa flow, Hilo side of Allied Aggregates quarry, alt $111 \mathrm{~m}$, Hilo quad $\left(19^{\circ} 38.56^{\prime} \mathrm{N}, 155^{\circ} 02.89^{\prime}\right.$ W). Coll 1978 by J P Lockwood. Comment (JPL): 1 of youngest prehistoric flows in Hilo quad. Date appears much too old, and indicates that such amorphous material (precipitated tars?) cannot give reliable dates. 
W-3841.

Charcoal root fragments from Pahala ash, overlain by olivine-rich pahoehoe, probably same flow as W-3845, Puu Kuanene area, alt $793 \mathrm{~m}$, Wood Valley quad $\left(19^{\circ} 20.04^{\prime} \mathrm{N}, 155^{\circ} 24.49^{\prime} \mathrm{W}\right)$. Coll 1977 by P W Lipman. Comment (PWL): min date on late minor faulting along Kaoiki zone, and indicates how recently lava was able to flow to SE from summit of ML; flow moving in this direction would now be blocked by modern caldera walls.

W-4164.

$3750 \pm 70$

Charcoal fragments from base of aa flow with abundant small plagioclase phenocrysts, Puu Kinikini, alt 1996m, Keaiwa Reservoir quad $\left(19^{\circ} 16.04^{\prime} \mathrm{N}, 155^{\circ} 37.14^{\prime} \mathrm{W}\right)$. Coll 1977 by S Coleman and P W Lipman. Comment (PWL): older age than originally expected, as petrographically similar nearby flow, $\mathrm{W}-4022$, yielded age of $1860 \pm 70 \mathrm{yr}$. May indicate that this sector of rift zone erupted similar lavas over a period of at least $2000 \mathrm{yr}$.

W-4152.

$3800 \pm 90$

Charcoal fragments from thin lens of Pahala-like ash below transitional hummocky pahoehoe aa flow, coll from roadcut Hwy 11, alt $84 \mathrm{~m}$, Punaluu quad $\left(19^{\circ} 08.89^{\prime} \mathrm{N}, 155^{\circ} 30.97^{\prime} \mathrm{W}\right)$. Coll 1977 by P W Lipman. Comment (PWL): overlain by Ninole flow, W-4008 and $-4142(2300 \pm 60$; $2440 \pm 60)$ and underlain by pahoehoe flow, W-3813 (7950 \pm 110$)$

\section{W.4132.}

$3900 \pm 90$

Charcoal roots from thin lens of Pahala-like ash, below pahoehoe flow, Ninole Gulch, alt $372 \mathrm{~m}$, Punaluu quad $\left(19^{\circ} 09.65^{\circ} \mathrm{N}, 155^{\circ} 32.55^{\prime}\right.$ W). Coll 1977 by P W Lipman. Comment (PWL): uppermost of 3 such flows that underlie large Ninole aa flow in area.

W-4009.

$3900 \pm 80$

Carbonized wood, base of Punaluu aa flow, Punaluu Gulch, alt $488 \mathrm{~m}$, Punaluu quad $\left(19^{\circ} 11.36^{\prime} \mathrm{N}, 155^{\circ} 31.70^{\prime} \mathrm{W}\right)$. Coll 1977 by $\mathrm{P}$ W Lipman. Comment (PWL): overlain by W-4008 and $-4142(2300 \pm 60 ; 2440 \pm 60)$ and underlain by $\mathrm{W}-3813(7950 \pm 110)$.

W-3803.

$4050 \pm 50$

Organic-rich soil containing bits of charcoal, coll from cesspool, in Kaumana area of Hilo, Hilo quad (19 $\left.42.80^{\prime} \mathrm{N}, 155^{\circ} 07.09^{\prime} \mathrm{W}\right)$. Coll 1977 by J P Lockwood. Comment (JPL): soil is in discontinuous pockets at base of massive pahoehoe $2 \mathrm{~m}$ thick. Dates 1 of oldest surface flows in Hilo.

W-3855.

$4210 \pm 90$

Charcoal from Pahala Ash, below phenocryst-poor aa flow draped on Kaoiki fault scarp, Kilauea quad (19² 25.42' N, $155^{\circ} 20.37^{\prime}$ W). Coll 1977 by P W Lipman. Comment (PWL): overlies W-3840 (9500 \pm 140$)$, and is overlain by $\mathrm{W}-4006(290 \pm 70)$. 
W-3844.

$4340 \pm 80$

Charcoal fragments from soil zone below aa flow, draped over Kaoiki fault scarp, Halfway House, alt $921 \mathrm{~m}$, Wood Valley quad $\left(19^{\circ}\right.$ $\left.21.87^{\prime} \mathrm{N}, 155^{\circ} 23.26^{\prime} \mathrm{W}\right)$. Coll 1977 by P W Lipman. Comment (PWL): provides min age for faulting.

W-4135.

$5160 \pm 100$

Charcoal roots from thin layer of Pahala-like ash below pahoehoe flow, Ninole Gulch, alt $369 \mathrm{~m}$, Punaluu quad $\left(19^{\circ} 09.64^{\prime} \mathrm{N}, 155^{\circ} 32.55^{\prime}\right.$ W). Coll 1977 by P W Lipman. Comment (PWL): 2nd flow from top of pahoehoe sequence, underlying sample W-4132 (3900 \pm 90$)$.

W-3862.

$5650 \pm 90$

Charcoal fragments at top of Pahala-type ash, beneath young ML aa flow, SW of Mountain View, alt 528m, Mountain View quad $\left(19^{\circ} 32.57^{\prime}\right.$ N, $155^{\circ} 07.46^{\prime} \mathrm{W}$ ). Coll 1977 by P W Lipman. Comment (PWL): youngest ML flow in area; age indicates frequency of eruptive hazard to town of Mountain View.

W-3930.

$6160 \pm 110$

Charcoal root fragments in ash below transitional pahoehoe-aa ML flow, Alapai Gulch, alt $567 \mathrm{~m}$, Naalehu quad $\left(19^{\circ} 25.09^{\prime} \mathrm{N}, 155^{\circ} 35.70^{\prime} \mathrm{W}\right)$. Coll 1977 by P W Lipman. Comment (PWL): dates relatively young prehistoric flow erupted from 2400 to $2700 \mathrm{~m}$ level of SE rift zone.

W-4117.

$7300 \pm 100$

Carbonaceous soil from ash at base of top ML flow, bay S of Kahukupoko Point, alt $21 \mathrm{~m}$, Naalehu quad $\left(19^{\circ} 04.32^{\prime} \mathrm{N}, 155^{\circ} 33.43^{\prime} \mathrm{W}\right)$. Coll 1977 by P W Lipman. Comment (PWL): overlies W-4121 (13,210 \pm 190$)$; probably underlies W-856 (R, 1960, v 2, p 157) and W-2016 (R, 1970, v 12, p 333) which yielded ages of $3740 \pm 250$ and $3620 \pm 250$, respectively.

\section{W-3813.}

$7950 \pm 110$

Black carbonaceous soil at top of Pahala ash, Puehu quarry, alt $76 \mathrm{~m}$, Punaluu quad $\left(19^{\circ} 08.75^{\prime} \mathrm{N}, 155^{\circ} 30.97^{\prime} \mathrm{W}\right)$. Coll 1977 by P W Lipman and J P Lockwood. Comment (PWL): oldest surface flow in Punaluu area; provides indirect limit on rate of subsidence since Puehu littoral cone formed.

W-3853.

$8550 \pm 100$

Charcoal root fragments from soil zone below ML pahoehoe flow, interbedded with Pahala Ash, or reworked ash, Kaoiki Pali, alt $1122 \mathrm{~m}$, Kilauea quad $\left(19^{\circ} 25.18^{\prime} \mathrm{N}, 155^{\circ} 20.33^{\prime} \mathrm{W}\right)$. Coll 1977 by $\mathrm{P}$ W Lipman. Comment (PWL): provides younger limit on age of faulting and Pahala Ash in area.

W-4201.

$9170 \pm 100$

Charcoal roots in Pahala ash below phenocryst-poor pahoehoe flow, part of flow sequence from ML that laps out against eroded Ninole Hills, 
Upper Moaula Gulch, alt 652m, Punaluu quad (19० $12.44^{\prime}$ N, $155^{\circ} 32.28^{\prime}$ W). Coll 1978 by P W Lipman. Comment (PWL): date constrains recurrence rate for activity in Kau dist.

\section{W-3840.}

$9500 \pm 140$

Charcoal rootlets and carbonaceous soil from Pahala ash between 2 ML aa flows draped over Kaoiki fault scarp, Kaoiki Pali, alt $1144 \mathrm{~m}$, Kilauea quad $\left(19^{\circ} 25.09^{\prime} \mathrm{N}, 155^{\circ} 20.63^{\prime} \mathrm{W}\right)$. Coll 1977 by P W Lipman. Comment (PWL): min age for major movement on faults.

W-4160.

$10,290 \pm 150$

Charcoal roots in Pahala Ash below phenocryst-poor pahoehoe flow, Honuapu, alt $186 \mathrm{~m}$, Naalehu quad (19०06.02' N, $\left.155^{\circ} 33.56^{\prime} \mathrm{W}\right)$. Coll 1977 by P W Lipman. Comment (PWL): flow ponds against, and is not significantly offset by, major young-appearing NE-trending fault that extends from Naalehu to above Honuapu. Min age for last major faulting.

\section{W-4014.}

$10,820 \pm 90$

Charcoal roots in uppermost main Pahala ash, near tree molds in overlying pahoehoe flow sequence, Hilea gulch, alt $229 \mathrm{~m}$, Punaluu quad $\left(19^{\circ} 08.30^{\prime} \mathrm{N}, 155^{\circ} 32.93^{\prime} \mathrm{W}\right)$. Coll 1977 by P W Lipman. Comment (PWL): from cliff exposure, ca $25 \mathrm{~m}$ below Kipuka Nene flow, W-4012, $-4156,-4137(740 \pm 60 ; 740 \pm 60 ; 890 \pm 60)$, indicating recurrence rate in area.

\section{W-3487.}

$$
11,780 \pm 100
$$

Charcoal fragments in Pahala ash under intercalated basalt flow, W side of Kaalualu Bay, 9.2km NE of South Point, Ka Lae quad (18 $58.30^{\prime}$ N, 155 $\left.37.30^{\prime} \mathrm{W}\right)$. Coll 1976 by J P and B M Lockwood.

\section{W-4121.}

$$
13,210 \pm 190
$$

Carbonaceous soil from ash at base of middle flow sequence, bay $\mathrm{S}$ of Kahukupoko Point, alt $15 \mathrm{~m}$, Naalehu quad $\left(19^{\circ} 04.34^{\prime} \mathrm{N}, 155^{\circ} 33.42^{\prime}\right.$ W). Coll 1977 by P W Lipman. Comment (PWL): underlies W-4117 $(7300 \pm 90)$; overlies $\mathrm{W}-3935(31,100 \pm 900)$, indicating recurrence rate of lavas entering ocean in area.

\section{W-4019.}

$26,410 \pm 390$

Thin carbonaceous mat, scraped from base of pahoehoe flow of Ninole volcanic rocks, Hilea Gulch, alt $183 \mathrm{~m}$, Punaluu quad (19०08.32' $\left.\mathrm{N}, 155^{\circ} 32.88^{\prime} \mathrm{W}\right)$. Coll 1977 by P W Lipman. Comment (PWL): 1st carbon sample ever coll from Ninole volcanic rocks; preliminary $\mathrm{K}-\mathrm{Ar}$ ages from these rocks suggest ages of several 100,000 yr.

\section{W-3935.}

$$
31,100 \pm 900
$$

Charcoal roots in Pahala-like ash, at base of tree mold in overlying $\mathrm{ML}$ aa flow, base of sea cliff in bay $\mathrm{S}$ of point, Kahukupoko Point, alt $1.5 \mathrm{~m}$, Naalehu quad $\left(19^{\circ} 04.27^{\prime} \mathrm{N}, 155^{\circ} 33.40^{\prime} \mathrm{W}\right)$. Coll 1977 by $\mathrm{P} \mathrm{W}$ Lipman. Comment (PWL): lowest flow sequence in sea-cliff, overlain by W-4121 (13,210 \pm 90$)$. Min date for main Pahala Ash. 
W-4128.

$31,400 \pm 1600$

Charcoal fragments in ash, beneath small pahoehoe lobe of mainly aa flow, along prominent main ash bed exposed along Pali Kapu O Keoua, Kealakekua scarp, alt 4.6m, Honaunau quad $\left(19^{\circ} 29.11^{\prime} \mathrm{N}, 155^{\circ}\right.$ $55.88^{\prime}$ W). Coll 1978 by P W Lipman. Comment (PWL): limiting age for major Kealakekua fault and rate of lava accumulation in area.

\section{Kilauea series}

W-3486.

Modern

Wood (Ohia) from fallen tree in kipuka, overlying pahoehoe, alt

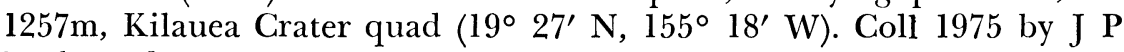
Lockwood.

W-3481.

Modern

Wood (Koa) from fallen tree in Ohia-Koa forest, in aa and ash, alt

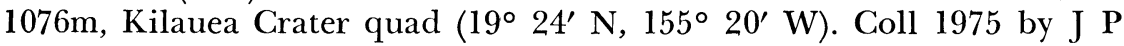
and B M Lockwood.

W-3480.

Wood (Ohia) from kipuka of older aa lava surrounded by finer, fresher aa of Keamoku flow, alt $1079 \mathrm{~m}$, Kilauea Crater quad $\left(19^{\circ} 24^{\prime} \mathrm{N}\right.$, $\left.155^{\circ} 20^{\prime} \mathrm{W}\right)$. Coll 1975 by J P and B M Lockwood.

W-3483.

Wood (Ohia) from standing tree in Ohia-Koa forest, alt $1076 \mathrm{~m}$, Kilauea Crater quad $\left(19^{\circ} 24^{\prime} \mathrm{N}, 155^{\circ} 20^{\prime} \mathrm{W}\right)$. Coll 1975 by J P and B M Lockwood.

W-3485.

Wood (Ohia) from standing tall giant, in Ainahou Ranch pahoehoe, alt $920 \mathrm{~m}$, Makaopuhi Crater quad (19 $\left.21^{\prime} \mathrm{N}, 155^{\circ} 14^{\prime} \mathrm{W}\right)$. Coll 1975 by J P Lockwood.

General Comment (JPL): W-3480, -3481, -3483, -3485, -3486 were taken from innermost heart wood of largest, and oldest, trees found growing on prehistoric lavas near summit of Kilauea volcano. It was hoped this would place realistic min ages on underlying lava flows. Their uniformly young ages likely attest to very high growth rate of trees in this tropical environment, although re-equilibration of carbon isotopes in "dead" heartwood is possibility.

W-2999.

Modern

Humus layer in yellow ash, overlying pahoehoe flow of pre-historic age, Thurston Lava Tube, Volcano quad (19² $\left.24^{\prime} 18^{\prime \prime} \mathrm{N}, 155^{\circ} 14^{\prime} 53^{\prime \prime} \mathrm{W}\right)$. Coll 1973 by R I Tilling. Comment (JPL): possibly within Keanakakoi or younger ash deposit. 
W.2970.

Wood from Kapoho Cone, Kilauea $\mathrm{E}$ rift, Kapoho quad $\left(19^{\circ} 30.1^{\prime} \mathrm{N}\right.$, $154^{\circ} 51.8^{\prime} \mathrm{W}$ ). Coll 1973 by R I Tilling. Comment (RIT): indicates another late prehistoric eruptive event on Kilauea's lower $\mathrm{E}$ rift.

W-4184.

$<\mathbf{2 0 0}$

Charcoal underlying tube-fed pahoehoe in large kipuka, Ainahou Ranch, alt $765 \mathrm{~m}$, Makaopuhi Crater quad $\left(19^{\circ} 19.80^{\prime} \mathrm{N}, 155^{\circ} 12.99^{\prime} \mathrm{W}\right)$. Coll 1978 by J P Lockwood. Comment (JPL): 1 of youngest prehistoric flows on Kilauea's $\mathrm{N}$ flank and may date Hawaiian oral legend of Pele and Kamapua. ${ }^{14} \mathrm{C}$ date initially seemed anomalously young, but subsequent field work indicates that flow overlies lithic ash probably of AD 1790.

W-3468.

Charcoal in root mold where lava flowed into crack, underlying fountain-fed pahoehoe, Kilauea $\mathrm{E}$ rift zone, Makaopuhi quad $\left(19^{\circ} 22.45^{\prime}\right.$ $\left.\mathrm{N}, 155^{\circ} 08.17^{\prime} \mathrm{W}\right)$. Coll 1975 by $\mathrm{R} \mathrm{T}$ Holcomb. Comment (RTH): younger date than expected; if valid, gives max age for Napau crater.

W-3467.

Charcoal underlying fountain-fed pahoehoe flow, from SE side of small crater SE of Napau, Kilauea E rift zone, Makaopuhi quad (19 $\left.22.45^{\prime} \mathrm{N}, 155^{\circ} 8.17^{\prime} \mathrm{W}\right)$. Coll 1975 by R T Holcomb.

W-3886.

Charcoal fragments in weathered brown ash, underlying Keanakakoi Ash, from pit, Kipuka $\mathrm{Ki}$, alt $1283 \mathrm{~m}$, Kilauea quad $\left(19^{\circ} 26.80^{\prime} \mathrm{N}, 155^{\circ}\right.$ 19.15' W). Coll 1977 by $\mathrm{P}$ W Lipman. Comment (PWL): indicates Keanakokoi Ash may all have been erupted in 1790, or at most within $\sim 100$ yr before. Dates last major explosive activity at Kilauea.

W-3937.

$<\mathbf{2 0 0}$

Charcoal from tree mold where lava ponded rapidly against base of fault scarp, Wood Valley quad (19० $\left.17.62^{\prime} \mathrm{N}, 155^{\circ} 24.04^{\prime} \mathrm{W}\right)$. Coll 1977 by R T Holcomb. Comment (RTH): sampled flow belongs to complex of flows that accumulated to form Kealaalea Hills-Puu Kou-Yellow Cone shield and extended as far $\mathrm{S}$ as the Kamooalii lava flows, latest prehistoric complex resulting from sustained eruption, analogous to Mauna Iki complex of 1919-20, on Kilauea's SW rift zone.

\section{W-3938.}

$210 \pm 60$

Charcoal in tree mold, $3 \mathrm{~m}$ from eruptive fissure, Wood Valley quad $\left(19^{\circ} 15.58^{\prime} \mathrm{N}, 155^{\circ} 24.62^{\prime} \mathrm{W}\right)$. Coll 1977 by $\mathrm{R} \mathrm{T}$ Holcomb. Comment (RTH): sampled flow is oldest of at least 3 short-lived but voluminous eruptions of similar age from parallel fissures seaward of Great Crack on SW rift zone of Kilauea. These flows predate prehistoric Kamooalii flows and flows of Red Cone but are apparently youngest flood-type eruptions older than eruption flood of 1823. 
W-3881.

$260 \pm 70$

Charcoal root fragments in ash below phenocryst-poor Kilauea pahoehoe in Fern Acres subdivision, alt $415 \mathrm{~m}$, Mountain View quad $\left(19^{\circ} 32.78^{\prime} \mathrm{N}, 155^{\circ} 05.89^{\prime} \mathrm{W}\right)$. Coll 1977 by $\mathrm{P}$ W Lipman. Comment (PWL): dates youngest flow from Kilauea Iki area to move this far $\mathrm{E}$ ( $20 \mathrm{~km}$ from caldera).

W-4162.

$310 \pm 70$

Charcoal roots from ash at base of Kilauea pahoehoe flow, coll along SE side of Wung quarry, alt $784 \mathrm{~m}$, Volcano quad $\left(19^{\circ} 28.29^{\prime} \mathrm{N}, 155^{\circ}\right.$ $10.16^{\prime}$ W). Coll 1977 by P W Lipman. Comment (PWL): dates most NE flow of Kilauea, where Kilauea lavas lap onto ML shield.

\section{W-3811.}

$330 \pm 60$

Charcoal fragments from Kilauea pahoehoe flow, beneath young prehistoric ML pahoehoe flow exposed in gully, Pahala quad $\left(19^{\circ} 13.03^{\prime} \mathrm{N}\right.$, $\left.155^{\circ} 27.70^{\prime} \mathrm{W}\right)$. Coll 1977 by J P Lockwood and P W Lipman. Comment (JPL): dates young-appearing pahoehoe flow from Kilauea SW rift zone. Unexpected recent age suggests that much of Kilauea SW rift activity is rather young.

\section{W-3849.}

$340 \pm 60$

Carbonaceous soil and charcoal at base of pyroclastic sequence, underlying basal reticulite of Keanakakoi Ash, in pit in Olaa Forest Reserve, depth $50 \mathrm{~cm}$, Wright $\mathrm{Rd}$, alt $1177 \mathrm{~m}$, Kilauea quad $\left(19^{\circ} 28.03^{\prime} \mathrm{N}, 155^{\circ}\right.$ 15.09' W). Coll 1977 by P W Lipman and J P Lockwood. Comment $(\mathrm{PWL})$ : with W-3886 $(<200)$ confirms young age of Keanakakoi Ash.

\section{W-3870.}

$350 \pm 60$

Organic-rich clay with charcoal fragments near base of Keanakakoi Ash, Kilauea quad $\left(19^{\circ} 26.63^{\prime} \mathrm{N}, 155^{\circ} 15.11^{\prime} \mathrm{W}\right)$. Coll 1977 by J P Lockwood. Comment (PWL): with samples from same horizon, W-3886 $(<200)$ and W-3849 (340 \pm 60$)$, indicates entire Keanakakoi Ash was erupted in 1790 , or at most, within $\sim 100$ yr prior. $\mathrm{W}-3999(730 \pm 80)$ is a Kilauea pahoehoe flow directly below Keanakakoi Ash, $1 \mathrm{~km}$ SE on Wright Rd.

\section{W-3800.}

$350 \pm 150$

Carbonaceous debris under prehistoric aa flow, depth $2 \mathrm{~m}$, from cesspool excavation, Puna, Pahoa South quad $\left(19^{\circ} 26.23^{\prime} \mathrm{N}, 154^{\circ} 56.11^{\prime}\right.$ W). Coll 1976 by J P Lockwood. Comment (JPL): indicates another undated prehistoric flow and attests to frequent eruptive activity along $\mathbf{E}$ rift zone.

\section{W-3018.}

$450 \pm 60$

Charcoal from Structure C, Wahaula Heiau, Kalapana quad $\left(19^{\circ}\right.$ 19.6' N, $\left.155^{\circ} 01.9^{\prime} \mathrm{W}\right)$. Coll 1972 by E J Ladd; subm by $\mathrm{R} I$ Tilling. Comment (RIT): date is min for prehistoric Kilauea flow, on which Wahaula Heiau was constructed. 
W-3842.

Charcoal fragments from soil below phenocryst-poor pahoehoe flow from Kilauea along base of Kaoiki Pali, in old quarry, Kilauea SW rift, alt $792 \mathrm{~m}$, Wood Valley quad $\left(19^{\circ} 19.98^{\prime} \mathrm{N}, 155^{\circ} 24.34^{\prime} \mathrm{W}\right)$. Coll 1977 by P W Lipman. Comment (PWL): geologically reasonable age for flow similar in appearance to W-3811 $(330 \pm 60)$ and W-3860 $(670 \pm 60)$. Overlies W-3841 (2950 \pm 80$)$.

\section{W-3941.}

$450 \pm 60$

Charcoal from top of ash layer beneath tube-fed pahoehoe flow partly filling old stream channel between 2 kipukas of ash-covered aa apparently derived from ML, Mountain View quad $\left(19^{\circ} 35.26^{\prime} \mathrm{N}, 155^{\circ} 01.01^{\prime}\right.$ W). Coll 1977 by R T Holcomb. Comment (RTH): tube-fed pahoehoe is apparently extensive unit on NE flank of Kilauea and is significantly older than other surficial Kilauea flows. Date helps determine time at which Kilauea grew sufficiently high to spill lava flows extensively over E subaerial edifice of ML.

\section{W-3860.}

$670 \pm 60$

Charcoal in Pahala Ash, below thin pahoehoe, 1 of last major prehistoric Kilauea SW rift flows, coll along small gully, tributary to Kaalaala Gulch, alt 399m, Pahala quad (19 $13.94^{\prime}$ N, $155^{\circ} 27.58^{\prime}$ W). Coll 1977 by P W Lipman. Comment (PWL): together with W-3811 $(330 \pm 60)$ and $\mathrm{W}-3842(450 \pm 60)$ indicates youth of surface lavas along much of Kilauea SW rift.

\section{W-3999.}

$730 \pm 80$

Carbonaceous soil containing small charcoal fragments from beneath toe of pahoehoe flow, alt $1134 \mathrm{~m}$, Volcano quad $\left(19^{\circ} 27.06^{\prime} \mathrm{N}, 155^{\circ} 14.32^{\prime}\right.$ W). Coll 1977 by P W Lipman. Comment (PWL): this flow immediately underlies Keanakakoi Ash and represents margin of thick widespread multiple-flow accumulation on NE side of Kilauea caldera.

W-3856.

$1040 \pm 70$

Carbonaceous clay and ash at base of pyroclastic base-surge sequence, just $\mathrm{N}$ of Kilauea volcano, in pit in Olaa Forest Reserve, depth $80 \mathrm{~cm}$, Wright Rd, alt $1177 \mathrm{~m}$, Kilauea quad $\left(19^{\circ} 28.03^{\prime} \mathrm{N}, 155^{\circ} 15.09^{\prime} \mathrm{W}\right)$. Coll 1977 by P W Lipman and J P Lockwood. Comment (PWL): dates last major explosive eruption from Kilauea, prior to Keanakakoi Ash; may correlate with Uwekatiuna Ash.

W-3827.

$1130 \pm 60$

Charcoal beneath Kilauea basalt flow, overlying Hilina Series rocks exposed in Poliokeawe Pali, Kau Desert quad $\left(19^{\circ} 18.10^{\prime} \mathrm{N}, 155^{\circ} 18.38^{\prime}\right.$ W). Coll 1977 by J P Lockwood. Comment (JPL): 1 of oldest surface flows on Kilauea. 
W.4119.

$1800 \pm 80$

Carbonaceous baked ash containing charred root fragments beneath aa flow mantling Pahala Ash on rim of Kukalauula Pali, Naliikakani Point quad $\left(19^{\circ} 14.80^{\prime} \mathrm{N}, 155^{\circ} 21.15^{\prime} \mathrm{W}\right)$. Coll 1977 by R T Holcomb. Comment (RTH): this aa flow may be 1 of oldest Kilauea lava flows now at surface; age helps define interval required for most of surface of Kilauea to become mantled by new lava flows.

W-3859.

$2080 \pm 70$

Carbonaceous clay and ash, underlying base-surge sequence, same location as W-3856, depth $130 \mathrm{~cm}$. Comment (PWL): this date correlates well with 2 other dates from Wright Rd, $340 \pm 60$, W-3849, and $1040 \pm$ $70, \mathrm{~W}-3856$, and gives a local recurrence interval for dangerous explosive activity from Kilauea.

W-3885.

$2160 \pm 70$

Charcoal around base of tree mold, in crystal-poor Kilauea pahoehoe, coll from quarry on Wung Ranch, alt $786 \mathrm{~m}$, Volcano quad $\left(19^{\circ} 28.28^{\prime} \mathrm{N}\right.$, $155^{\circ} 10.17^{\prime} \mathrm{W}$ ). Coll 1977 by P W Lipman. Comment (PWL): same flow as $\mathrm{W}-4162(310 \pm 70)$; age discrepancy not yet understood, but younger age is geologically more reasonable.

\section{W-3831.}

$3480 \pm 80$

Charcoal beneath Kilauea basalt flow, overlying rocks of Hilina Volcanics, exposed in Poliokeawe Pali, Kau Desert quad $\left(19^{\circ} 18.11^{\prime} \mathrm{N}, 155^{\circ}\right.$ $\left.18.37^{\prime} \mathrm{W}\right)$. Coll 1977 by J P Lockwood. Comment (JPL): flow is overlain by $\mathrm{W}-3827(1130 \pm 60)$, and underlain by $\mathrm{W}-3798(4820 \pm 200)$. W-3798 is underlain by $\mathrm{W}-3809(10,680 \pm 130)$. Together, these 4 flows give recurrence interval for this one area.

\section{W-3884.}

$3610 \pm 60$

Charcoal in ash below olivine-rich pahoehoe flow, Pahala vent, alt $177 \mathrm{~m}$, Pahala quad $\left(19^{\circ} 12.73^{\prime} \mathrm{N}, 155^{\circ} 28.16^{\prime} \mathrm{W}\right)$. Coll 1977 by $\mathrm{P}$ W Lipman. Comment (PWL): flow was erupted from lowermost large vent on Kilauea's SW rift. Vent fissure follows fractures of Kaoiki fault zone.

W-4177.

$3690 \pm 70$

Charcoal beneath pahoehoe exposed in trench, Puna Sugar Field \#20, SE from Keaau, alt $142 \mathrm{~m}$, Mountain View quad $\left(19^{\circ} 34.93^{\prime} \mathrm{N}, 155^{\circ}\right.$ 01.82' W). Coll 1978 by J P Lockwood. Comment (RTH): should be among oldest Kilauea flows now at surface.

W-3798.

$4820 \pm 90$

Carbonized charcoal tree fern (Hapu'u) from ash directly above lava dated by W-3809 $(10,680 \pm 130)$, coll in new scar formed during 1975 earthquake, Hilina Pali, Kau Desert quad (19²8.16 $\left.\mathrm{N}, 155^{\circ} 18.34^{\prime} \mathrm{W}\right)$. Coll 1976 by J P Lockwood. Comment (JPL): dates overlying lava flow, which in turn is overlain by W-3831 $(3480 \pm 80)$. 
W-3873.

Organic-rich ash containing charcoal fragments from uppermost level of thick Pahala Ash sequence in W side of Kaena Bihopa Arroyo,

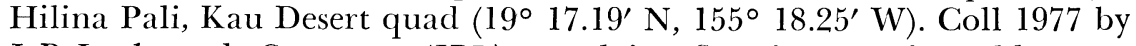
J P Lockwood. Comment (JPL): overlying flow is unconformably overlain by glassy prehistoric pahoehoe that forms dike-like fracture-filling sheets downslope.

\section{W-3809.}

$10,680 \pm 130$

Charcoal beneath basalt and ash in upper Hilina Pali sec, coll from new scar formed during 1975 earthquake, Kau Desert quad $\left(19^{\circ} 18.16^{\prime} \mathrm{N}\right.$, $155^{\circ} 18.34^{\prime} \mathrm{W}$ ). Coll 1976 by J P Lockwood. Comment (JPL): with 4820 $\pm 90, \mathrm{~W}-3798$, date accumulation of $1.2 \mathrm{~m}$ of Pahala Ash.

\section{W-3801.}

Charcoal from top of Pahala Ash sec on Puu Kaone, Kau Desert quad $\left(19^{\circ} 16.27^{\prime} \mathrm{N}, 155^{\circ} 17.17^{\prime} \mathrm{W}\right)$. Coll 1976 by M Easton. Comment (JPL): date conflicts with 17,360 \pm 650 (W-905, R, 1961, v 3, p 94) which was coll from lower part of Pahala ash at this locality.

\section{W-3814.}

$22,800 \pm 340$

Charcoal exposed in fresh outcrop created by massive slumping during 1975 earthquake, Puu Kaone, Kau Desert quad $\left(19^{\circ} 16.25^{\prime} \mathrm{N}, 155^{\circ}\right.$ 17.33' W). Coll 1976 by M Easton. Comment (JPL): agrees well with $22,500 \pm 300, \mathrm{~W}-3801$, from same horizon, $500 \mathrm{~m}$ to $\mathrm{W}$. These 2 dates suggest that $17,360 \pm 650$ date $(\mathrm{W}-905, \mathrm{R}, 1961, \mathrm{v} 3$, p 94) from below same sec may be too young. Comment (ES): date of W-905 may be in error, due to small sample size which necessitated large dilution for analysis.

\section{REFERENCES}

Lockwood, J P, and Lipman, P W, 1979, Recovery of charcoal for ${ }^{14} \mathrm{C}$ dating from beneath lava-lessons from Hawaii: Jour Volc and Geothermal Res, $v 4$.

Peterson, D W, 1967, Geologic map of Kilauea Crater Quadrangle: US Geol Survey GQ Map 667.

Rubin, Meyer and Alexander, Corinne, 1960, US Geological Survey radiocarbon dates V: Radiocarbon, v 2, p 129-185.

Rubin, Meyer and Berthold, S M, 1961, US Geological Survey radiocarbon dates VI: Radiocarbon, v 3, p 86-98.

Sullivan, B M, Spiker, E, and Rubin, M, 1970, US Geological Survey radiocarbon dates XI: Radiocarbon, v 12, p 319-334. 